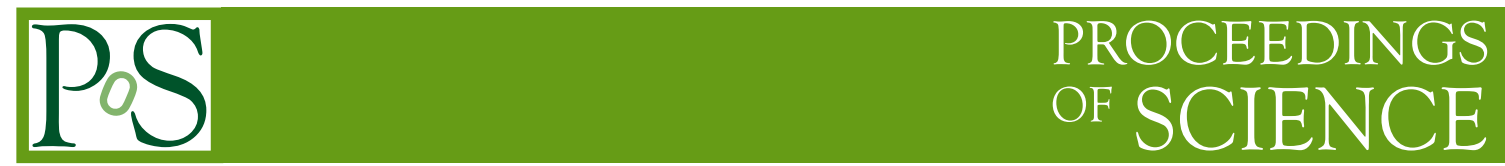

\title{
Collectivity in small colliding systems
}

\section{Piotr Bożek*}

AGH University of Science and Technology, Faculty of Physics and Applied Computer Science, al. Mickiewicza 30, 30-059 Krakow, Poland E-mail: piotr.bozek@fis.agh.edu.pl

Collective expansion of the matter created in ultrarelativistic heavy-ion collisions indicates the formation of strongly interacting quark-gluon plasma. Significant final state interactions, generating a collective flow are expected and observed in experiments with a small projectile. I present a comparision of the results of viscous hydrodynamic models for small colliding systems with experimental data. These observations rise to questions about the nature of the matter created in small systems and the limitations of the hydrodynamic model.

7th International Conference on Physics and Astrophysics of Quark Gluon Plasma

1-5 February, 2015

Kolkata, India

${ }^{*}$ Speaker. 

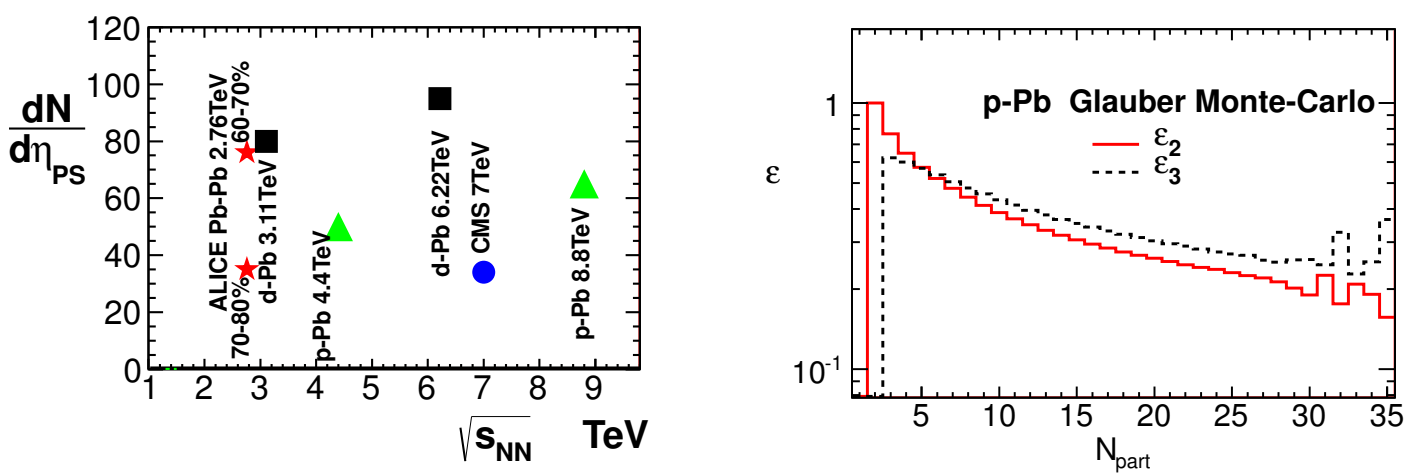

Figure 1: (left panel) Charged particle density in different colliding systems at LHC energies. (right panel) Ellipticity and triangularity in p-Pb collisions from the Glauber Monte Carlo model (from [16]).

\section{Introduction}

The existance of a collective expansion stage of the fireball created in heavy-ion collisions at energies of $200 \mathrm{GeV}$ and $2760 \mathrm{GeV}$ is supported by a number of experimental observation $[1,2,3,4,5,6,7,8]$. The strongest argument in favor of the collective expansion comes from the observation of the azimuthal asymmetry of the spectra, i.e. the elliptic and triangular flows.

On the other hand, it has been expected that in collisions with a small projectile, such as in $\mathrm{p}-\mathrm{Pb}$ collisions, final state interactions are negligible [9]. $\mathrm{p}-\mathrm{Pb}$ collisions and d-Au collisions could serve as a reference system without collectivity. Interesting phenomena based on the physics of gluon saturation could be observed in such collisions [10, 11, 12, 13, 14, 15]. However, simple extrapolation of the multiplicity from peripheral $\mathrm{Pb}-\mathrm{Pb}$ to $\mathrm{p}-\mathrm{Pb}$ collision, and estimates of the source size in the Glauber Monte Carlo model indicate that large energy densities can be reached in small colliding systems. Quantitative estimates in the hydrodynamic model [16] have shown that substantial elliptic and triangular flow is produced in $\mathrm{p}-\mathrm{Pb}$ collisions at LHC energies. In the following we give a short review of the comparison of the scenario with collective expansion with experimental observations and the LHC and RHIC.

\section{Why collective expansion is expected in $\mathrm{p}-\mathrm{Pb}$ collisions}

The energy density in the center of the fireball formed in relativistic heavy-ion collisions allows the formation of the quark-gluon plasma. The dense matter confined in the small source feels large gradients of pressure, resulting in a very effective acceleration of the matter. In the left panel of Fig. 1 is shown the expected charge particle density in several colliding systems. The multiplicity in peripheral $\mathrm{Pb}-\mathrm{Pb}$, central $\mathrm{p}-\mathrm{Pb}$ and the most violent $\mathrm{p}-\mathrm{p}$ collisions is comparable. Moreover, one expects that in $\mathrm{p}-\mathrm{Pb}$ or $\mathrm{p}-\mathrm{p}$ collisions the size of the interaction region, where the energy is deposited, is smaller than in $\mathrm{Pb}-\mathrm{Pb}$ interactions. Large energy density and small size of the fireball implies the possibility of a very rapid, explosive expansion of the source.

The most convincing experimental observation of the collective expansion is based on the asymmetry of the flow. For that to happen a significant asymmetry of the source in the transverse 

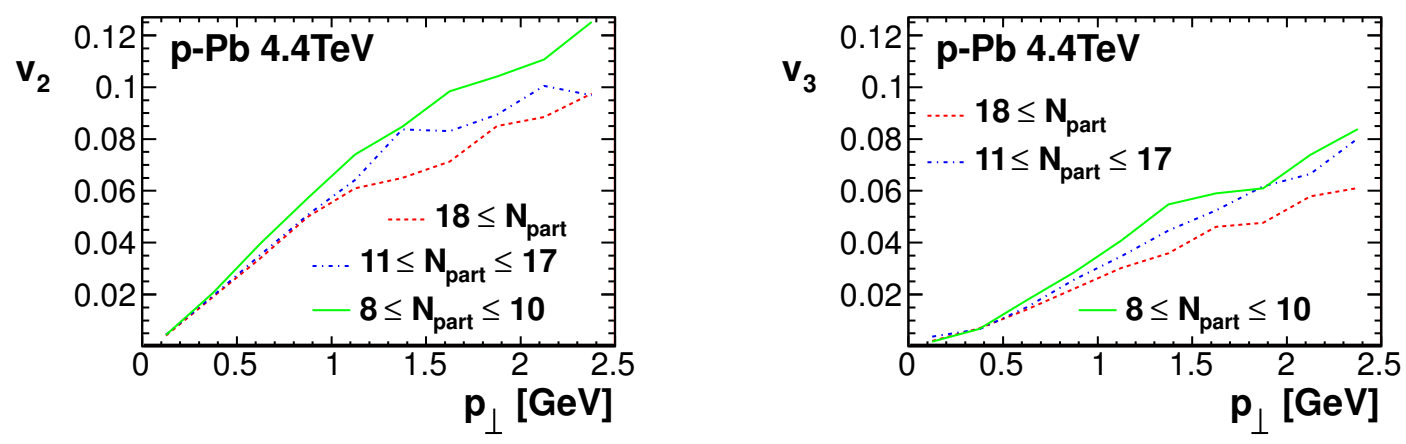

Figure 2: Elliptic (left panel) and triangular (right panel) flow of charged particles in p-Pb collisions predicted by the hydrodynamic model (from [16]).

plane is necessary. In the right panel of Fig. 1 we plot the ellipticity and the triangularity of the initial fireball calculated in the Glauber Monte Carlo model. In collisions of protons with a large nucleus the asymmetry originates entirely from event by event fluctuation of the distribution of participating nucleons. As the number of participants is moderate, such fluctuations are large and give rise to a significant deformation of the fireball.

We have noticed that the two most important assumptions necessary for the generation of the azimuthally asymmetric collective flow in $\mathrm{p}-\mathrm{Pb}$ collisions are meet. Performing a simulation of the $3+1$-dimensional viscous hydrodynamic model in this system allows to estimate the final flow. The expected elliptic an triangular flow is noticeable, and could easily be identified in events of multiplicity of around 100. All heavy-ion experiments at the LHC have observed azimuthally asymmetric flow in $\mathrm{p}-\mathrm{Pb}$ collisions $[17,18,19]$. In the next sections we give more detailed arguments for the collective expansion in $\mathrm{p}-\mathrm{Pb}, \mathrm{d}-\mathrm{Au}$ or $\mathrm{He}-\mathrm{Au}$ collisions $[20,21]$.

\section{Signs of collectivity}

\subsection{Elliptic and triangular flow}

The hydrodynamic expansion generates azimuthally asymmetric transverse flow. Several calculations have been performed for a number of different small colliding systems at RHIC and LHC energies [16, 24, 25, 26, 27, 28, 29, 30, 31, 32]. All calculations based on Glauber model initial conditions give a large elliptic and triangular flow of produced particles. Quantitatively the calculations give the right magnitude of the flow. In the region of the validity of hydrodynamic models calculations give the correct $p_{\perp}$ dependence of the flow coefficients as well (Fig. 3). The centrality dependence of the flow coefficients $v_{2}$ and $v_{3}$ is the result of the interplay of two opposite trends, the reduction of the initial anisotropy for more central collisions (Fig. 1, right panel) and the increase of the hydrodynamic response as the system gets larger. Detailed modeling must involve fluctuation of the energy deposition from each participant, qualitatively the experimental centrality dependence of the flow coefficient can be reproduced [29]. 

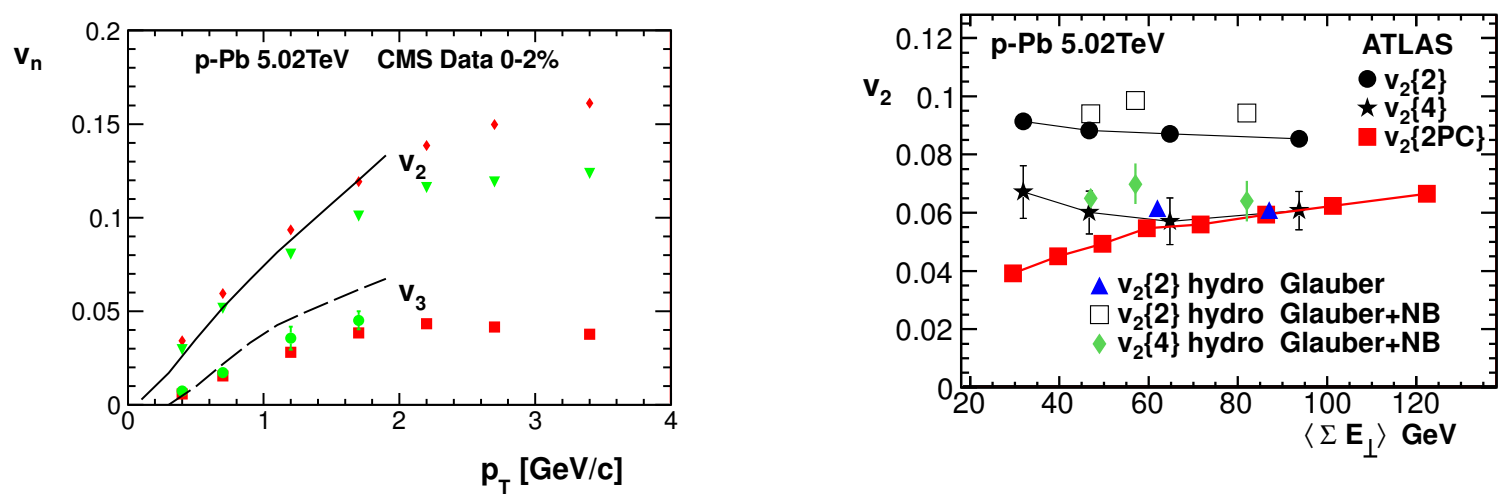

Figure 3: (left panel) Elliptic and triangular flow of charged particles, data from the CMS Collaboration compared to hydrodynamic model results (from [22]). (right panel) Elliptic flow of charged particles measured by the ATLAS Collaboration [23] using three different methods, also are shown the predictions of the hydrodynamic model for $v_{2}$ using the second and fourth cumulant (from [24]).

\subsection{Higher cumulants}

It is essential to distinguish the correlation due to collective flow from other sources of correlations, such as resonance decays or jets. For two particle correlations the contribution from resonance decays scales as the inverse of the multiplicity. In small multiplicity events the identification of small flow signal becomes difficult. To remove the contribution from non-flow correlation estimates of flow coefficients from higher cumulants can be used [33, 34]. In the Glauber Monte Carlo model of one can estimate all the moments of the initial eccentricity [35]. For p-Pb collisions all moments are expected to be of similar magnitude, only the second moment is larger. Exactly the same trend is observed in experiments [23,36,37,38], where $v_{2}\{2\}>v_{2}\{4\} \simeq v_{2}\{6\} \simeq v_{2}\{8\}$.

The analysis of the probability distribution of initial eccentricities in $\mathrm{p}-\mathrm{Pb}$ collisions gives predictions on specific relations between flow coefficients from different cumulants [39]. These relations are consistent with experimental observations. In summary, the observation of flow measured with higher cumulants gives confidence to the collective expansion scenario in $\mathrm{p}-\mathrm{Pb}$ collisions. Moreover, detailed relations between the magnitude of the flow coefficients of different order can be traced to analogous relations between initial eccentricity cumulants.

\subsection{Small deformed systems}

The eccentricity of the fireball in $\mathrm{p}-\mathrm{Pb}$ collisions is generated by fluctuations. This essential parameter of the hydrodynamic model can be constrained by using small deformed nuclei. In collisions involving a deutron projectile the fireball has a strong deformation [16]. Events with large deformation can be selected by the event multiplicity (Fig. 4, left panel). In such events the deutron hits the larger nucleus with its elongated side. Moreover, the predicted magnitude of the deformation of the fireball is less sensitive to the details of the model of the initial state. The idea can be generalized to collisions with a ${ }^{3} \mathrm{He}$ nucleus as a projectile, in that case events with enhanced triangularity would occur [27]. 

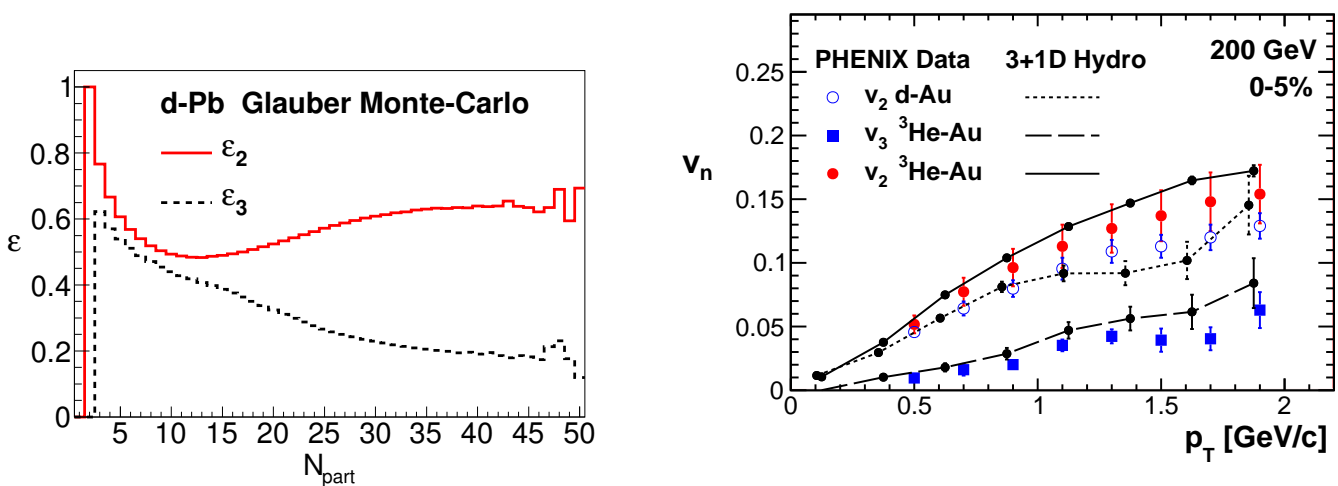

Figure 4: (left panel) Large elliptic deformation in d-Pb collisions, predicted in the Glauber Monte Carlo model (from [16]). Elliptic and triangular flow in $\mathrm{He}-\mathrm{Au}$ and d-Au collisions, hydrodynamic model compared to PHENIX Collaboration data [40] (from [41]).
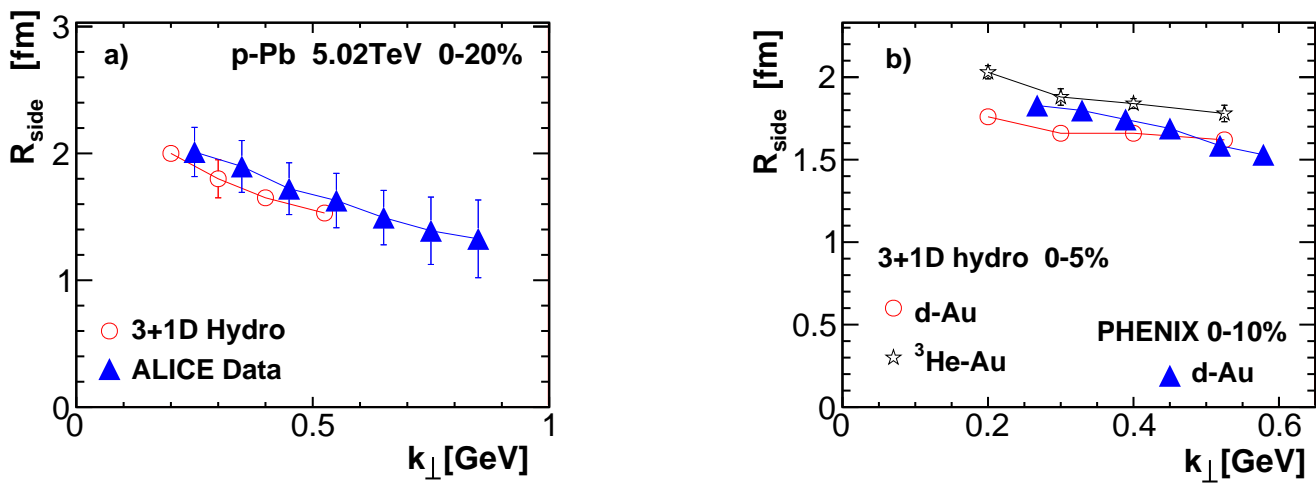

Figure 5: $R_{\text {side }}$ as a function of the pair transverse momentum for $\mathrm{p}-\mathrm{Pb}$ collisions (left panel) calculated in the hydrodynamic model, data from the ALICE Collaboration [43], and for d-Au and He-Au collisions (right panel), hydrodynamic model results [44, 41] compared to data from the PHENIX Collaboration [21].

Hydrodynamic simulations of collisions with a small deformed nucleus predict a large elliptic flow, in line with the large initial ellipticity of the fireball [27, 41]. The final triangular flow in ${ }^{3} \mathrm{He}$-Au collisions is enhanced, although not as much as naively expected, because most of the configurations in He-Au collisions have a moderate triangularity [42]. It is striking that the calculations are in a very good agreement with experimental observations [20, 21, 40]. It shows that the observed flow asymmetry results from a response to the deformation in the initial geometry.

\subsection{Interferometry radii}

The size of the source formed in collisions with a small projectile is an essential parameter. Within hydrodynamic models the size measured by interferometry correlations is related to the size of the homogeneity region at freeze-out [45]. The presence of the transverse collective flow at freeze-out implies a characteristic dependence of the interferometry radii on the average pair 

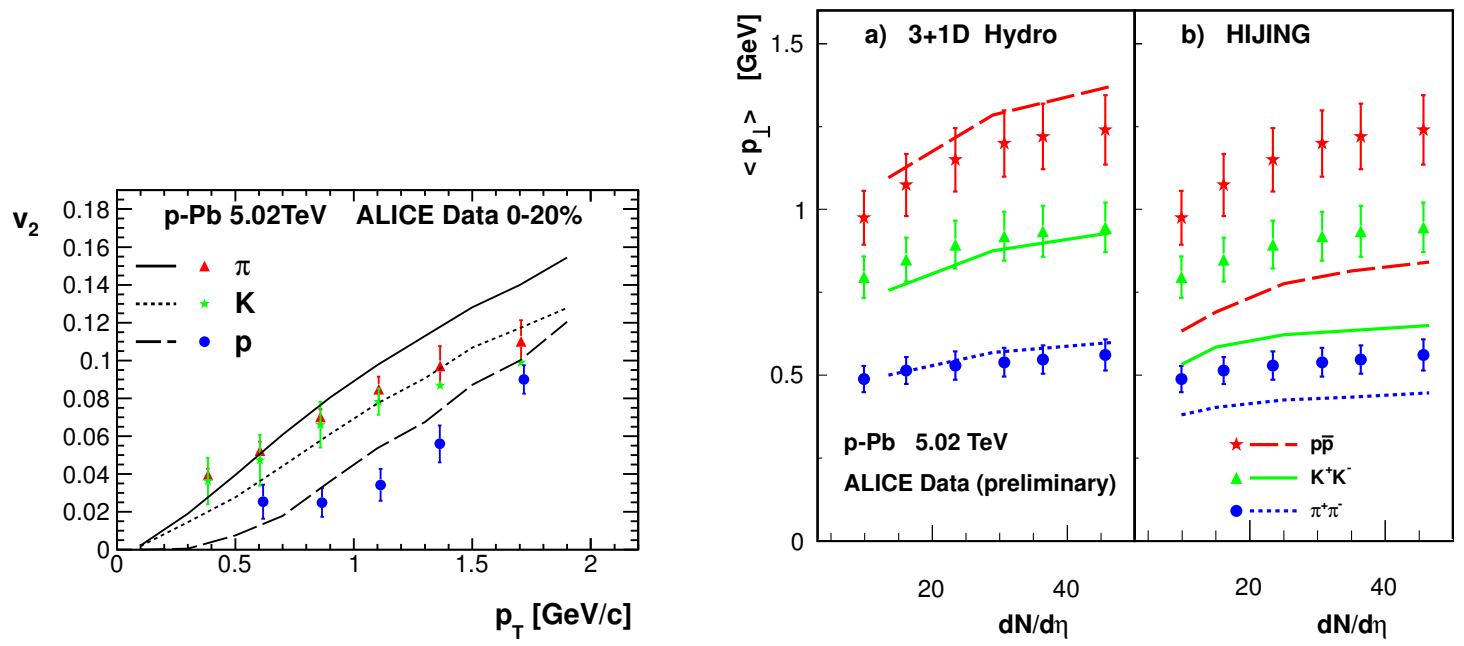

Figure 6: (left panel) Elliptic flow of identified particles in p-Pb collisions for the hydrodynamic model compared to ALICE Collaboration data [48]. (right panel) Average transverse momentum of identified particles in $\mathrm{p}-\mathrm{Pb}$ collisions form the HIJING model, from the hydrodynamic model and data from the ALICE Collaboration [49] (from [22]).

momentum. Pion pairs emitted with large, almost colinear momenta, originate from the same fluid element. The collimation effect makes the effective size of this fluid element to shrink as the average pair momentum increases.

Experimental results for $\mathrm{p}-\mathrm{p}, \mathrm{p}-\mathrm{Pb}$ and $\mathrm{Pb}-\mathrm{Pb}$ collisions at the LHC show, that the size of the source in $\mathrm{p}-\mathrm{Pb}$ collisions is in between the size for $\mathrm{p}-\mathrm{p}$ and $\mathrm{Pb}-\mathrm{Pb}$ systems $[46,43]$. Three interferometry radii can be calculated and compared to experimental results [47, 44, 30]. Good agreement is obtained, both for $\mathrm{p}-\mathrm{Pb}$ and $\mathrm{d}-\mathrm{Au}$ [21] collisions (Fig. 5). The strong azimuthal deformation of the transverse flow in d-Au collisions raises the possibility to observe the angular dependence of the radii using azimuthally sensitive interferometry [44]. In summary, the observation of interferometry radii as expected from model calculations, shows that the size of the fireball is well predicted. The observed dependence of the interferometry radii on pair momentum is consistent with the presence of a strong radial flow in the system.

\subsection{Mass splitting of the elliptic flow}

Particle emission from a fluid element at freeze-out is determined by kinematics and in particular by the particle mass. A generic prediction of the hydrodynamic models is that the elliptic flow of a particle depends on its mass. Heavy particles, such as protons, have a smaller elliptic flow at small momenta than light pions. The mass splitting of the elliptic flow coefficient as a function of $p_{\perp}$ would occur if collective expansion is present in small colliding systems [22, 27, 28]. A mass splitting in the elliptic flow coefficient is observed in p- $\mathrm{Pb}$ [48] collisions (Fig. 6, left panel), and in d-Au collisions [21].

\subsection{Mass hierarchy of transverse flow}

The azimuthal asymmetry of the flow requires that the transverse flow is present in the system. 
The transverse flow leads to a mass hierarchy of the average transverse momenta of emitted particles. Heavy particles pick up a larger transverse momentum from the transverse flow velocity of the fluid cell. In the right panel of Fig. 6 is shown the centrality dependence of the average transverse momentum for pions, kaons and protons. In the experimental data [49] and in the hydrodynamic model $[22,50]$ the mass hierarchy of the transverse flow is clearly visible, whereas the HIJING models shows a much weaker mass hierarchy. The effect of the transverse flow can be seen directly in the mass and centrality dependence of the transverse momentum spectra of identified particles [51, 49].

\section{Conclusions and prospects}

First, the energy density in the source is large. Second, the mean free path in a low viscosity plasma at the temperature of $500 \mathrm{MeV} \simeq 0.3 \mathrm{fm}$ is significantly smaller than the systems size $\simeq 2 \mathrm{fm}$. These estimates justify the use of the hydrodynamic model for the dynamics of the fireball in small colliding systems. The hydrodynamic model predicts a significant transverse collective flow in $\mathrm{p}-\mathrm{Pb}, \mathrm{d}-\mathrm{Au}$, and $\mathrm{He}-\mathrm{Au}$ collisions.

Formally, the range of applicability of hydrodynamics is limited to system close to local equilibrium with small gradients of the density and flow [31]. Certainly, these conditions are not meet at the early stage of the collision, where pressure becomes asymmetric in the longitudinal and transverse directions. However, the effect of this pressure asymmetry on the final observables is small $[52,53,54]$.

Hydrodynamics ceases to be applicable at freeze-out, where the interaction rate drops. If this transition region is narrow it can be approximated as a sharp freeze-out surface. It turns out that in $\mathrm{p}-\mathrm{Pb}$ collisions the size of this region can become relatively large, as demonstrated by simulations in cascade models $[55,56]$. Nevertheless the hydrodynamic model gives similar results as the cascade model, which means that the Cooper-Frye prescription with a sharp freeze-out surface still works effectively in small systems.

Future investigations of collectivity in small systems will go in two directions. First, the conditions where collectivity switches off should be found. We expect it to happen in small enough systems (p-p) or at lower energies. Second. the hydrodynamic response in small asymmetric colliding systems provides a mapping of the initial conditions into the final flow. It offers a unique opportunity to study the early dynamics in the collision and the mechanism of the energy deposition in space-time.

\section{References}

[1] I. Arsene et al. (BRAHMS), Nucl. Phys. A757, 1 (2005)

[2] B. B. Back et al. (PHOBOS), Nucl. Phys. A757, 28 (2005)

[3] J. Adams et al. (STAR Collaboration), Nucl. Phys. A757, 102 (2005)

[4] K. Adcox et al. (PHENIX Collaboration), Nucl. Phys. A757, 184 (2005)

[5] K. Aamodt et al. (ALICE Collaboration), Phys. Rev. Lett. 105, 252302 (2010)

[6] K. Aamodt et al. (ALICE Collaboration), Phys. Lett. B696, 328 (2011) 
[7] G. Aad et al. (ATLAS), Phys. Rev. Lett. 105, 252303 (2010)

[8] S. Chatrchyan et al. (CMS), Phys. Rev. C84, 024906 (2011)

[9] C. Salgado, J. Alvarez-Muniz, F. Arleo, N. Armesto, M. Botje, et al., J.Phys. G39, 015010 (2012)

[10] K. Dusling and R. Venugopalan, Phys. Rev. Lett. 108, 262001 (2012)

[11] K. Dusling and R. Venugopalan, Phys. Rev. D 87, 054014 (2013)

[12] K. Dusling and R. Venugopalan, Phys. Rev. D 87, 051502 (2013)

[13] Y. V. Kovchegov and D. E. Wertepny, Nucl.Phys. A906, 50 (2013)

[14] A. Kovner and M. Lublinsky, Int. J. Mod. Phys. E Vol. 22 (2013)

[15] K. Dusling and R. Venugopalan, Phys. Rev. D87, 094034 (2013)

[16] P. Bożek, Phys. Rev. C85, 014911 (2012)

[17] S. Chatrchyan et al. (CMS Collaboration), Phys. Lett. B718, 795 (2013)

[18] B. Abelev et al. (ALICE Collaboration), Phys. Lett. B719, 29 (2013)

[19] G. Aad et al. (ATLAS Collaboration), Phys. Rev. Lett. 110, 182302 (2013)

[20] A. Adare et al. (PHENIX Collaboration), Phys. Rev. Lett. 111, 212301 (2013)

[21] N. Ajitanand et al. (PHENIX), Nucl.Phys. A931, 1082 (2014)

[22] P. Bożek, W. Broniowski, and G. Torrieri, Phys. Rev. Lett. 111, 172303 (2013)

[23] G. Aad et al. (ATLAS Collaboration), Phys. Lett. B725, 60 (2013)

[24] P. Bożek and W. Broniowski, Phys. Rev. C88, 014903 (2013)

[25] A. Bzdak, B. Schenke, P. Tribedy, and R. Venugopalan, Phys. Rev. C87, 064906 (2013)

[26] G.-Y. Qin and B. Müller, Phys. Rev. C89, 044902 (2014)

[27] J. Nagle, A. Adare, S. Beckman, T. Koblesky, J. O. Koop, et al., Phys.Rev.Lett. 113, 112301 (2014)

[28] K. Werner, M. Bleicher, B. Guiot, I. Karpenko, and T. Pierog, Phys. Rev. Lett. 112, 232301 (2014)

[29] I. Kozlov, M. Luzum, G. Denicol, S. Jeon, and C. Gale(2014), arXiv:1405.3976 [nucl-th]

[30] P. Romatschke, Eur. Phys. J. C75, 305 (2015)

[31] H. Niemi and G. S. Denicol(2014), arXiv:1404.7327 [nucl-th]

[32] C. Shen, J. F. Paquet, G. S. Denicol, S. Jeon, and C. Gale(2015), arXiv:1504.07989 [nucl-th]

[33] N. Borghini, P. M. Dinh, and J.-Y. Ollitrault, Phys. Rev. C63, 054906 (2001)

[34] N. Borghini, P. M. Dinh, and J.-Y. Ollitrault, Phys.Rev. C64, 054901 (2001)

[35] A. Bzdak, P. Bożek, and L. McLerran, Nucl.Phys. A927, 15 (2014)

[36] S. Chatrchyan et al. (CMS Collaboration), Phys. Lett. B724, 213 (2013)

[37] V. Khachatryan et al. (CMS), Phys. Rev. Lett. 115, 012301 (2015)

[38] B. B. Abelev et al. (ALICE), Phys. Rev. C90, 054901 (2014)

[39] L. Yan and J.-Y. Ollitrault, Phys. Rev. Lett. 112, 082301 (2014)

[40] A. Adare et al. (PHENIX)(2015), arXiv:1507.06273 [nucl-ex] 
[41] P. Bozek and W. Broniowski, Phys. Lett. B747, 135 (2015)

[42] P. Bożek and W. Broniowski, Phys.Lett. B739, 308 (2014)

[43] J. Adam et al. (ALICE), Phys. Rev. C91, 034906 (2015)

[44] P. Bożek, Phys.Rev. C90, 064913 (2014)

[45] S. Akkelin and Y. Sinyukov, Phys. Lett. B356, 525 (1995)

[46] B. B. Abelev et al. (ALICE), Phys. Lett. B739, 139 (2014)

[47] P. Bożek and W. Broniowski, Phys. Lett. B720, 250 (2013)

[48] B. B. Abelev et al. (ALICE Collaboration), Phys. Lett. B726, 164 (2013)

[49] B. B. Abelev et al. (ALICE Collaboration), Phys.Lett. B728, 25 (2014)

[50] K. Werner, B. Guiot, I. Karpenko, and T. Pierog, Phys. Rev. C89, 064903 (2014)

[51] S. Chatrchyan et al. (CMS), Eur. Phys. J. C74, 2847 (2014)

[52] J. Vredevoogd and S. Pratt, Phys. Rev. C79, 044915 (2009)

[53] P. Bożek and I. Wyskiel-Piekarska, Phys. Rev. C83, 024910 (2011)

[54] R. Ryblewski and W. Florkowski, Phys. Rev. C85, 064901 (2012)

[55] A. Bzdak and G.-L. Ma, Phys.Rev.Lett. 113, 252301 (2014)

[56] L. He, T. Edmonds, Z.-W. Lin, F. Liu, D. Molnar, et al.(2015), arXiv:1502.05572 [nucl-th] 